\title{
Ewing's Sarcoma/Primitive Neuroectodermal Tumor of the Uterine Corpus
}

\author{
Eung-Seok Lee • Won Hwangbo ${ }^{1}$ Insun Kim ${ }^{1}$ \\ Department of Pathology, Korea University Ansan Hospital, Ansan; 'Department of Pathology, Korea University Anam Hospital, Seoul, Korea
}

Ewing's sarcoma (ES)/primitive neuroectodermal tumor (PNET) is defined as a round cell sarcoma that shows varying degrees of neuroectodermal differentiation. The term PNET was first introduced by Hart and Earle in $1973 .{ }^{1}$ Cases of ES/PNET in the female genital tract are rare. Now we report a case of ES/ PNET arising in the uterine body which was confirmed by all studies performed, including morphologic, immunohistochemical, ultrastructural, and cytogenetic examinations.

\section{CASE REPORT}

A 23-year-old Korean woman presented with irregular vaginal bleeding and low abdominal pain. Ultrasonography and magnetic resonance imaging of the pelvis revealed a huge intramural mass of the uterus, measuring $8.4 \mathrm{~cm}$ in diameter. Bilateral adnexal abnormality or significant lymphadenopathy was not identified. Total abdominal hysterectomy, bilateral salpingo-oophorectomy, and lymph node dissections were done.

Grossly, the uterus was slightly enlarged, measuring $6 \times 13 \times 8$ $\mathrm{cm}$. On opening the uterus, there was a mass arising from the anterior wall of the uterus, bulging out into the endometrial cavity with an area of ulceration on the endometrium. The cut surface of the uterus showed an unencapsulated but relatively well-circumscribed intramural tumor, measuring $9 \times 7.5 \mathrm{~cm}$ (Fig. 1). The tumor showed a homogeneous gray-tan, solid, and fish-fleshy appearing cut surface with no conspicuous necrosis

\section{Corresponding Author}

Eung-Seok Lee, M.D.

Department of Pathology, Korea University Ansan Hospital, 123 Jeokgeum-ro, Danwon-gu, Ansan 425-707, Korea

Tel: +82-31-412-5323, Fax: +82-31-412-5324, E-mail: a9604@chollian.net

Received: July 16, 2014 Revised: September 21, 2014

Accepted: October 13, 2014 or hemorrhage. The tumor abutted the endometrium and serosal surface of the uterus. Both ovaries and salpinges were grossly unremarkable without any enlargement or tumor identified.

Microscopically, the tumor was composed of relatively uniform small round-to-oval neoplastic cells arranged in a diffuse sheet or solid nesting pattern of growth with intervening fibrous septa throughout the myometrium. The tumor invaded the endometrium focally but did not involve the serosal surface of the uterus. There were numerous areas of lymphatic tumor invasion and a metastatic tumor implant on the surface of the left ovary, but there was no evidence of metastatic tumor in the pelvic lymph nodes. The tumor cells had scanty cytoplasm with indistinct cytoplasmic border, round-to-oval nuclei of stippled chromatin pattern, and inconspicuous nucleoli (Fig. 2). Mitoses, apoptotic bodies, and focal areas of necrosis were frequently found (22/10 high power fields of mean mitotic count). Pseudorosettes were also frequently present but no malignant glandular areas were identified within or adjacent to the tumor.

On immunohistochemical examination the tumor cells showed diffuse strong positivity for cluster of differentiation 99 antigen (CD99) and neuron-specific enolase (NSE) in a membranous pattern and Friend leukemia virus integration 1 (FLI-1) in a nuclear pattern. The tumor cells were focally positive for vimentin but negative for c-kit, WT-1, CAM5.2, chromogranin, synaptophysin, CD56, CD10, CD3, CD20, leukocyte common antigen, desmin, and myogenin (Fig. 3). Electromicroscopic study showed a variable number of glycogen particles in a dispersed pattern and there were primitive intercellular and synaptic-like junctions. We also performed dual-color fluorescence in situ hybridization (FISH) analysis with a break-apart rearrangement probe. In normal cells, a combined two-signal pattern is seen, reflecting intact regions; we found separated single green 

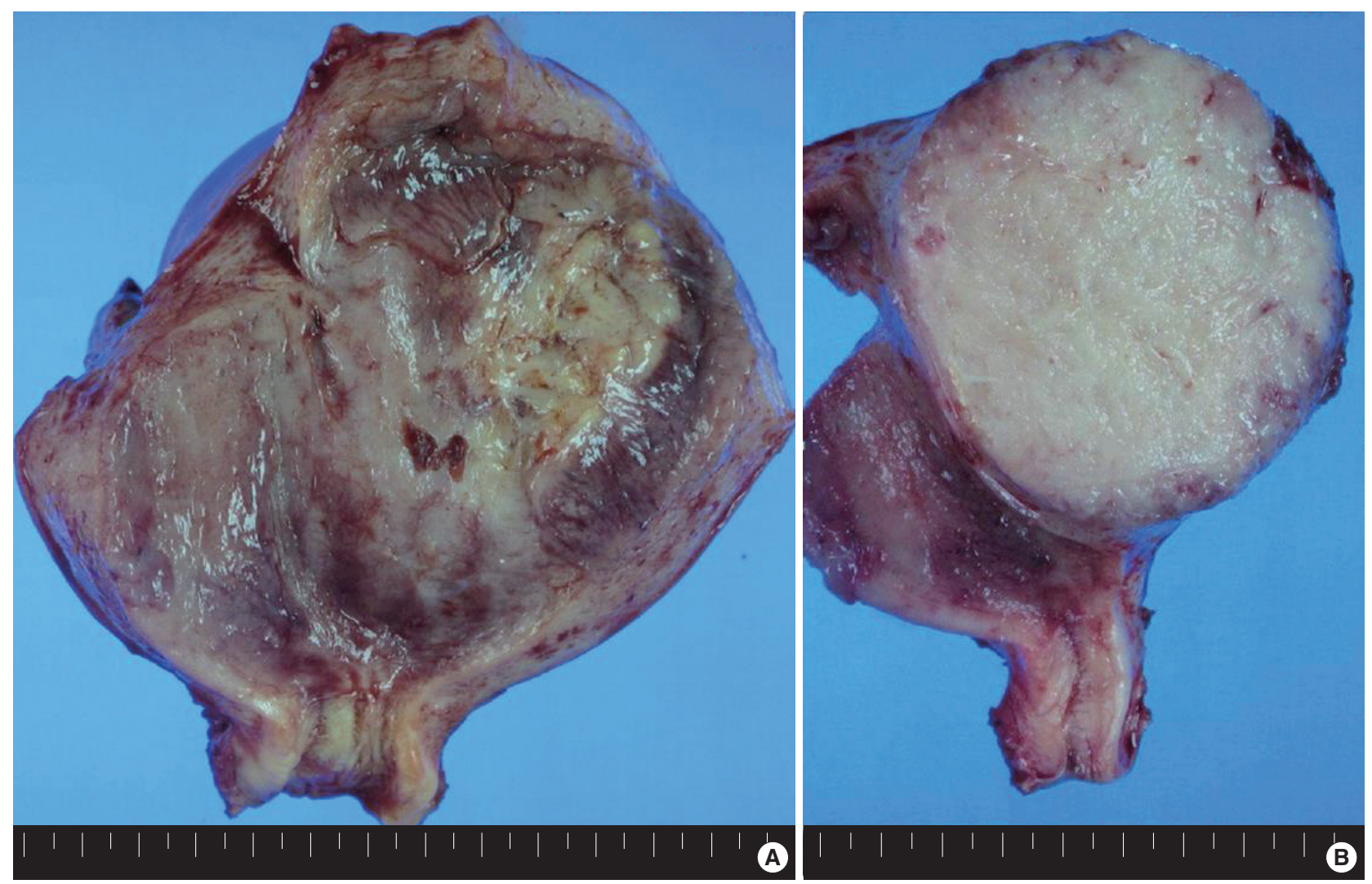

Fig. 1. (A) The uterus is slightly enlarged, measuring $6 \times 13 \times 8 \mathrm{~cm}$. On opening the uterus, there is a mass arising from the anterior wall of the uterus, bulging out into the endometrial cavity with an area of ulceration on the endometrium. (B) The cut surface of the uterus shows an unencapsulated but relatively well-circumscribed intramural tumor, measuring $9 \times 7.5 \mathrm{~cm}$. The tumor shows a homogeneous gray-tan, solid, and fish-fleshy appearing cut surface with no conspicuous necrosis or hemorrhage. The tumor abuts the endometrium and serosal surface of the uterus.
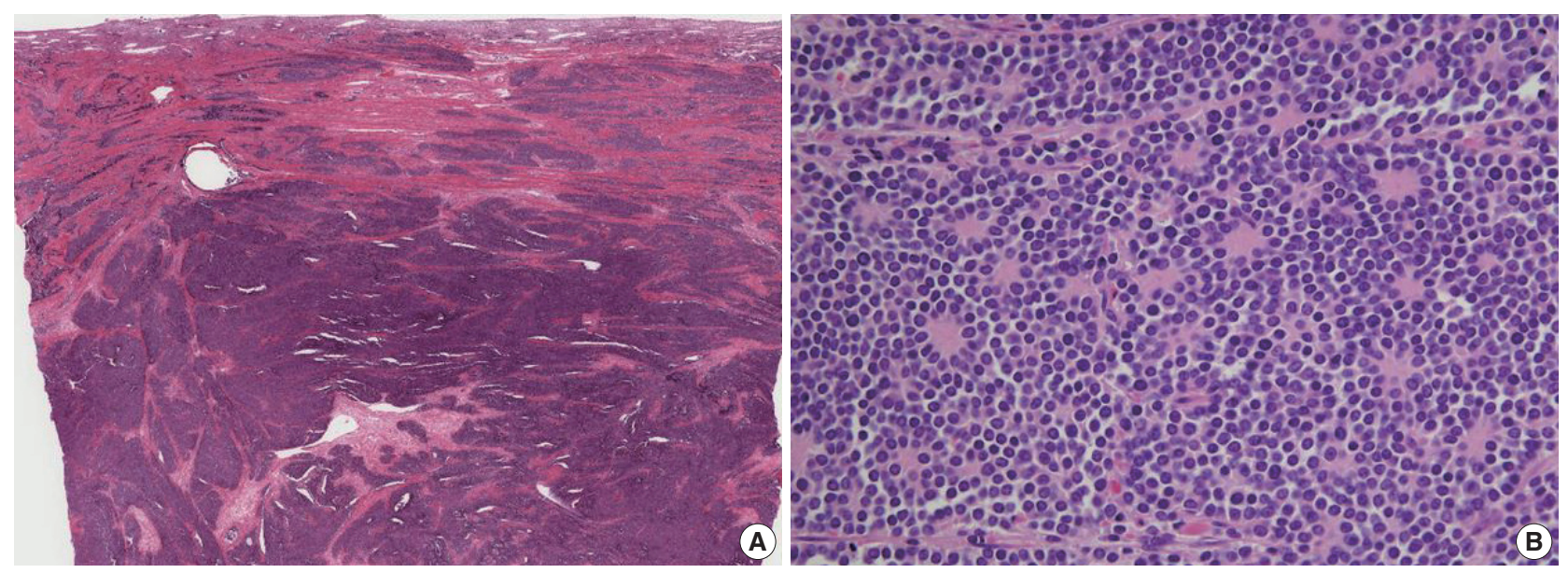

Fig. 2. (A) The tumor is composed of relatively uniform small round-to-oval neoplastic cells and arranged in a diffuse sheet or solid nesting pattern of growth with intervening fibrous septa throughout the myometrium. The tumor invades the endometrium focally but does not involve the serosal surface of the uterus. (B) The tumor cells have scant cytoplasm with an indistinct cytoplasmic border, round-to-oval nuclei of stippled chromatin pattern, and inconspicuous nucleoli. Pseudorosettes are also frequently present but no malignant glandular areas are identified within or adjacent to the tumor. 

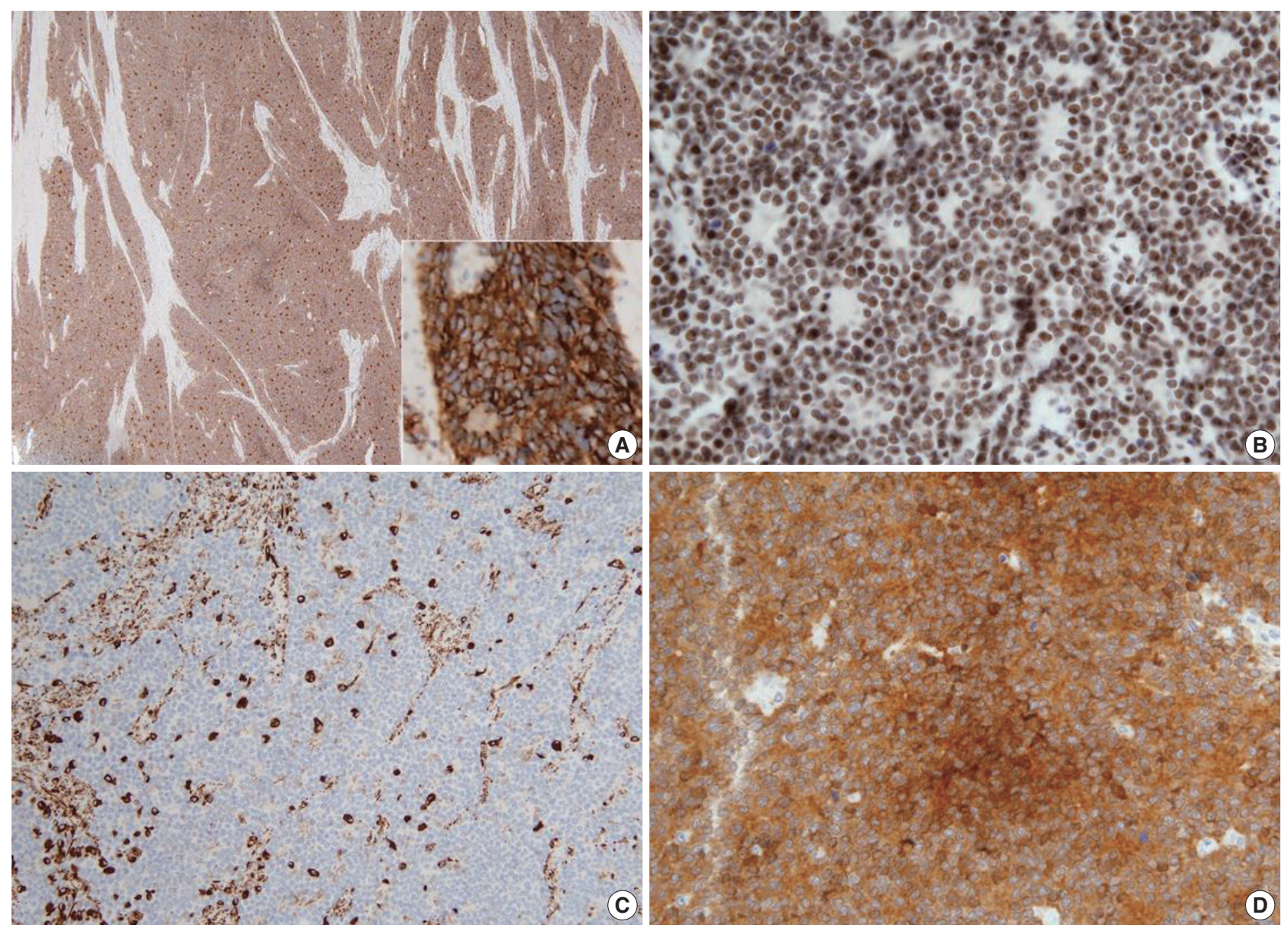

Fig. 3. The tumor cells show diffuse strong positivity for CD99 (A) and neuron-specific enolase (D) in a membrane pattern and FLI-1 (B) in a nuclear pattern. The tumor cells are focally positive for vimentin (C).

and single orange signal patterns and identified rearrangement in the tumor. On the basis of the FISH results, we performed reverse transcription polymerase chain reaction (RT-PCR) and confirmed the products of EWS-FLI1 fusion transcript with $330 \mathrm{bp}$.

\section{DISCUSSION}

ES/PNET is defined as a round cell sarcoma that shows varying degrees of neuroectodermal differentiation. The term PNET was first introduced by Hart and Earle in 1973 to describe a group of small round cell tumors that appeared to be derived from fetal neuroectodermal cells with variable degrees of neuroectodermal differentiation. ${ }^{1}$ ES and PNET are regarded as two extremes of a morphologic spectrum of the same tumor entity. Their classification depends on the degree of differentiation of their neuroectodermal components. ES with no evidence of neuroectodermal differentiation lies at one end of the spectrum and
PNET with clear evidence of neuroectodermal differentiation at the other. ES/PNET shows characteristic signature translocations involving the ES gene (EWSR1, Ewing sarcoma breakpoint region 1) at 22q12.2 and various erythroblastic transformation specific (ETS)-family transcription factor genes, the most common of which is FLI1 at 11q24.1-q24.3. ${ }^{2}$ EWSR1 is a ubiquitously expressed gene encoding a multifunctional protein that regulates multiple cellular processes, while FLI-1 is a transcription factor that plays a role during vascular development and in lymphoid function. ${ }^{3}$ The resulting fusion genes act as an oncogenic transcription factor. Cases of ES/PNET in the female genital tract are rare. Such tumors are found in the ovary, ${ }^{4}$ vulva, ${ }^{5}$ vagina, ${ }^{6}$ cervix,${ }^{7}$ and uterine corpus. ${ }^{8}$

On the basis of microscopic examination the differential diagnosis of ES/PNET of the uterine corpus includes poorly differentiated endometrioid carcinoma, stromal sarcoma, small cell neuroendocrine carcinoma, and lymphoma, which are composed of sheets of cytologically malignant small cells with little or no 
evidence of glandular or squamous differentiation. Small cell neuroendocrine carcinomas and lymphomas make developing a differential diagnosis difficult because they show immunohistochemical overlap as well as morphologic similarities with ES/ PNET. Histologically, small cell neuroendocrine carcinomas tend to grow in sheets of small cells having nuclei with stippled "salt and pepper" chromatin, absence of nucleoli, and nuclear molding of adjacent cells. Although rosettes and pseudorosettes, positive staining for chromogranin, synaptophysin, and NSE, and ultrastructural dense-core granules may be observed in both small cell carcinomas and ES/PNET, the former is usually immunohistochemically negative for MIC-2 and FLI-1, which are relatively specific markers for ES/PNET. The absence of lymphoid markers virtually excludes a diagnosis of lymphoma. Ultrastructural investigation may be helpful, since lymphoma shares no features with ES/PNET, which usually demonstrates neural differentiation, dense-core granules, and glycogen accumulation. Endometrioid carcinomas virtually always contain focal areas of glandular differentiation with positivity for epithelial markers. Stromal sarcomas are composed of cells that closely resemble non-neoplastic endometrial stroma and have a prominent vascular pattern of spiral arteriole-like spaces and mild nuclear atypia with low mitotic index. Stromal sarcoma can be excluded by areas of marked nuclear atypia with frequent mitotic figures and the absence of the typical vascular pattern in ES/ PNET. The absence of CD10 and estrogen and progesterone receptors also helps to make a diagnosis of ES/PNET.

MIC-2 and FLI-1 are very useful markers for the diagnosis of ES/PNET, as they are expressed in nearly all ES/PNET. ${ }^{8}$ In addition, ES/PNET tumor cells are positive for vimentin and may focally express NSE, chromogranin, synaptophysin, and S-100. In our case, the tumor cells were diffusely strongly positive for MIC-2 and FLI-1, and focally positive for vimentin. Ultrastructural features are helpful in making the diagnosis of ES/PNET. Ultrastructurally, neural differentiation, including neurosecretory granules and neurite-like cytoplasmic processes, can be recognized in ES/PNET. ${ }^{8}$ Glycogen accumulation in the cytoplasm also can be seen in ES/PNET. All ultrastructural features of the above were present in our case. ES/PNET is also characterized by balanced chromosomal translocation of $\mathrm{t}(11 ; 22)$ (q24;q12), resulting in the production of the EWS-FLI1 fusion gene in approximately $85 \%$ of cases. Other translocations leading to the fusion of the EWS gene with one of several members of the ETS family of transcription factors have subsequently been identified, including $\mathrm{t}(21,22)(\mathrm{q} 22 ; \mathrm{q} 21)$ in $10 \%-15 \%$ of cases and $\mathrm{t}(7 ; 22), \mathrm{t}(17 ; 22)$ and $\mathrm{t}(2 ; 22)$ in less than $1 \%$ of cases.
Thus genetic analysis would be helpful when histologic and immunohistochemical examinations are not conclusive. ${ }^{8} \mathrm{Nu}-$ merous molecular techniques such as DNA- and RNA-based polymerase chain reaction, Southern blotting, and FISH have been used for detection of the EWS translocations associated with ES/PNET. Ideally, cytogenetic investigation for the assessment of such translocations on paraffin sections should include FISH and RT-PCR. ${ }^{2}$ RT-PCR is unique in its ability to identify both genes involved in the most common translocations encountered in ES/PNET. A variety of RT-PCR assays have been developed for the detection of EWS translocation products. ${ }^{2}$ Recently, EWSR1 (22q12), a dual-color, break-apart rearrangement probe using FISH analysis (Abbott Laboratories, Abbott Park, IL, USA), has been commercially available, and its diagnostic value in ES/PNET is well-established. ${ }^{9}$ However, due to the presence of the $\mathrm{t}(11 ; 22)$ translocation in other tumor types such as desmoplastic small round cell tumor, ${ }^{10}$ FISH results should be interpreted in light of the morphology and immunohistochemical profile.

At initial diagnosis, the tumor is often already at an advanced stage in most patients. In addition, there is no uniformity in the treatment of ES/PNET in the uterus. Total hysterectomy with bilateral salpingo-oophorectomy, with or without chemotherapy and/or radiotherapy, is the usual course of treatment provided. In this case, the patient underwent surgical resection and six courses of chemotherapy. The patient is still alive with no evidence of recurrence or metastasis after operation in December 2012, and chemotherapy with ifosfamide.

\section{Conflicts of Interest}

No potential conflict of interest relevant to this article was reported.

\section{REFERENCES}

1. Hart MN, Earle KM. Primitive neuroectodermal tumors of the brain in children. Cancer 1973; 32: 890-7.

2. Masoura S, Kourtis A, Kalogiannidis I, et al. Primary primitive neuroectodermal tumor of the cervix confirmed with molecular analysis in a 23-year-old woman: a case report. Pathol Res Pract 2012; 208: 245-9.

3. Truong AH, Ben-David Y. The role of Fli-1 in normal cell function and malignant transformation. Oncogene 2000; 19: 6482-9.

4. Kleinman GM, Young RH, Scully RE. Primary neuroectodermal tumors of the ovary: a report of 25 cases. Am J Surg Pathol 1993; 17: 764-78. 
5. Vang R, Taubenberger JK, Mannion CM, et al. Primary vulvar and vaginal extraosseous Ewing's sarcoma/peripheral neuroectodermal tumor: diagnostic confirmation with CD99 immunostaining and reverse transcriptase-polymerase chain reaction. Int J Gynecol Pathol 2000; 19: 103-9.

6. Liao X, Xin X, Lü X. Primary Ewing's sarcoma-primitive neuroectodermal tumor of the vagina. Gynecol Oncol 2004; 92: 684-8.

7. Tsao AS, Roth LM, Sandler A, Hurteau JA. Cervical primitive neuroectodermal tumor. Gynecol Oncol 2001; 83: 138-42.

8. Ren YL, Tang XY, Li T. Ewing sarcoma-primitive neuroectodermal tumor of the uterus: a clinicopathologic, immunohistochemical and ultrastructural study of one case. Arch Gynecol Obstet 2011; 283: 1139-43.

9. Mhawech-Fauceglia P, Herrmann F, Penetrante R, et al. Diagnostic utility of FLI-1 monoclonal antibody and dual-colour, break-apart probe fluorescence in situ (FISH) analysis in Ewing's sarcoma/primitive neuroectodermal tumour (EWS/PNET): a comparative study with CD99 and FLI-1 polyclonal antibodies. Histopathology 2006; 49: 569-75.

10. Sandberg AA, Bridge JA. Updates on the cytogenetics and molecular genetics of bone and soft tissue tumors: desmoplastic small roundcell tumors. Cancer Genet Cytogenet 2002; 138: 1-10. 